\title{
Rigetto anticorpo-mediato nel trapianto di rene: fisiopatologia, clinica e terapia
}

\author{
Aris Tsalouchos ${ }^{1}$, Maurizio Salvadori² \\ ${ }^{1}$ Azienda UsI Toscana Centro, S.O.S. Nefrologia e Dialisi, Ospedale SS Cosma e Damiano, Pescia, Pistoia - Italia \\ ${ }^{2}$ Professore di Nefrologia, già Direttore Nefrologia e Trapianto, Azienda Ospedaliero-Universitaria Careggi, Firenze - Italia
}

\begin{abstract}
Antibody-mediated rejection in kidney transplantation: pathophysiology, clinic and therapy
Introduction. Over the past two decades, our thinking has changed from considering rejection as primarily a T-cell mediated process to the realization that insufficient control of the humoral arm of a recipient's immune system is the factor primarily responsible for the allograft dysfunction and loss. Acute Antibody Mediated Rejection (ABMR) in kidney transplantation is a severe complication that frequently occurs after transplantation and is due either to pre-transplant Donor Specific Antibodies (DSAs) or to de novo DSAs. New techniques to detect DSAs in the recipient serum and advances in the assessment of graft pathology have allowed us to recognize this entity in recent years.

Methods. The treatment of ABMR is a multistep process consisting in the desensitization of the patients with preformed antibodies to prevent acute ABMR: in case of acute ABMR, the antibodies are removed from the serum and anti-B cells immunosuppressants are used.

Results and Discussion. Along with our knowledge on acute ABMR, a distinct entity has been recognized: the chronic AMBR. Chronic ABMR is a frequent cause of late graft dysfunction and is characterized by a typical histopathologic feature. The treatment is often difficult and new drugs are now tested to control the disease.
\end{abstract}

Keywords: Antibody-mediated rejections, Complement activation, Desensitization, Donor specific antibodies, Treatment of antibody-mediated rejection

II rigetto anticorpo-mediato rappresenta una causa importante di disfunzione del trapianto renale e di perdita dell'organo precoce o tardiva.

Per quanto, generalmente, il rigetto anticorpo-mediato (ABMR) avvenga come risposta immunitaria nei confronti degli antigeni HLA del donatore espressi sulle cellule endoteliali, il AMR può verificarsi anche con anticorpi diretti non contro gli antigeni del sistema HLA, come gli antigeni del gruppo sanguigno $A B-0$, o a causa di autoanticorpi diretti nei confronti dei recettori di tipo 1 dell'angiotensina II.

\section{Tipi di AMR}

Si possono distinguere almeno tre tipi di AMR.

Received: September 13, 2020

Accepted: September 18, 2020

Published online: October 9, 2020

Indirizzo per la corrispondenza:

Maurizio Salvadori

Azienda Ospedaliero-Universitaria Careggi

Largo Brambilla, 3

50134 Firenze - Italia

maurizio.salvadori1@gmail.com

\section{AMR iperacuto}

Avviene a causa di anticorpi preformati ad alto titolo nei confronti del donatore (DSA). Si verifica molto precocemente dopo il trapianto. È raro, perché tali anticorpi vengono generalmente rilevati con le tecniche di cross-match pretrapianto.

Si hanno arterite trans murale, edema interstiziale e grave necrosi corticale.

\section{AMR acuto}

È sovente dovuto ad anticorpi contro il donatore (DSA) che o sono preformati a basso titolo o si sviluppano dopo il trapianto (1).

II rigetto acuto umorale (AMR) si verifica nel 5-7\% di tutti i trapianti renali.

Si hanno un danno endoteliale con infiltrazione di neutrofili dei glomeruli e dei capillari peritubulari e un edema interstiziale con emorragie. L'identificazione del AMR è divenuta più agevole con il riconoscimento del C4d nelle biopsie.

\section{Rigetto cronico AMR}

Gli anticorpi possono anche mediare un rigetto cronico, che è caratterizzato istologicamente dalla glomerulopatia da 
trapianto (2). La glomerulopatia da trapianto è caratterizzata da espansione del mesangio, duplicazione della membrana basale e pluristratificazione della membrana basale dei capillari peritubulari. È, in genere, asintomatico nelle prime fasi, ma si possono avere proteinuria in range nefrosico, ipertensione e lenta ma progressiva disfunzione renale. La prevalenza della glomerulopatia da trapianto nelle biopsie protocollari varia dal $5 \%$ a un anno al $20 \%$ a 5 anni (3).

\section{Fisiopatologia}

Gli anticorpi coinvolti sono, in genere, anticorpi diretti contro gli antigeni di istocompatibilità. Tuttavia, meno frequentemente, sono coinvolti anticorpi diretti contro altri antigeni, come i MICA, i MICB, antigeni piastrinici e antigeni del sistema renina-angiotensina (4-6).

Il sistema maggiormente coinvolto è l'attivazione del complemento dovuta al complesso antigene-anticorpo, che porta alla formazione del complesso di attacco della membrana che determina il danno cellulare. Questo soprattutto si verifica a livello endoteliale. Si ha anche un'attivazione piastrinica con formazione di microtrombi. I prodotti complementari C3a e C5a amplificano, poi, il processo flogistico. Gli anticorpi possono anche mediare il danno attraverso vie indipendenti dal complemento, come la citotossicità mediata da anticorpi (ADCC). Questa è, in genere, mediata da cellule natural killer o da macrofagi (7).

\section{Diagnosi di AMR}

Secondo la classificazione di Banff (8), il rigetto AMR è definito da una triade che coinvolge la presenza di DSA, la positività al C4d alla biopsia e il danno istopatologico tipico del danno anticorpale.

II rigetto acuto mediato da anticorpi è classificato in tre tipi:

Classe I: presenza di necrosi tubulare acuta (NTA) con minimi segni di flogosi

Classe II: glomerulite, capillarità peritubulare e microtrombosi

Classe III: arterite

Il rigetto cronico mediato da anticorpi (cAMR) richiede la dimostrazione di C4d, DSA e almeno uno dei tipici aspetti del danno cronico glomerulare, come il doppio contorno della membrana basale, la pluristratificazione della membrana basale dei capillari peritubulari e la fibrosi interstiziale con atrofia tubulare.

\section{Colorazione del C4d}

Il C4d è un prodotto complementare che si forma dalla frammentazione del C4b in C4d e C4c. Si trova normalmente nei reni normali nel mesangio e nel polo vascolare, come espressione di un normale turn over del complemento. La sua presenza nei capillari glomerulari e/o nei capillari peritubulari è espressione di attività antigene-anticorpo con attivazione complementare a livello endoteliale ed è, quindi, espressione di attacco anticorpale (9).

La presenza di C4d a livello glomerulare in corso di AMR non è, tuttavia, costante, pur essendo significativa la sua presenza. Si possono avere AMR anche in assenza di C4d, il che ha dato origine al concetto di AMR C4d negativi (10).

\section{Trattamento}

Molto spesso, il trattamento si avvale di una combinazione di terapie (Fig. 1).

In effetti, molteplici trattamenti sono stati impiegati nella terapia del AMR, come indicato nella Tabella I.

\section{(a) Immunoglobuline endovena}

Probabilmente agiscono attraverso processi molteplici, come la neutralizzazione degli anticorpi fissanti il complemento, le alterazioni del complemento stesso e la modulazione del fattore Fc.

Vengono usate ad alte dosi $(2 \mathrm{~g} / \mathrm{kg}$ ) o a basse dosi (100 mg/kg) per sessione.

\section{(b) Plasmaferesi}

È efficace nel ridurre la carica anticorpale presente nel siero del ricevente, ma richiede l'uso di altre terapie atte a ridurre la produzione anticorpale. Viene effettuata a giorni alterni, scambiando un volume di plasma con albumina o plasma fresco congelato.

Effetti collaterali consistono in alterazioni della coagulazione spesso collegati al necessario impiego di anticoagulanti.

\section{(c) Immunoassorbimento con proteina A (IA)}

Non è usato negli Stati Uniti, ma, in uno studio europeo randomizzato e controllato (11), IA si è dimostrato particolarmente efficace nel controllare il AMR.

\section{(d) Rituximab}

II Rituximab (RTX) è un anticorpo monoclonale anti-CD20, che induce deplezione delle cellule $B$. Diversi studi hanno impiegato RTX nel trattamento del AMR (12-15).

I risultati sono stati positivi usando dosi differenti di RTX e associazioni farmacologiche differenti.

\section{(e) Bortezomib}

Il Bortezomib è un inibitore del proteasoma che induce una riduzione dell'attivazione del NF-kB nucleare, arresto del ciclo 


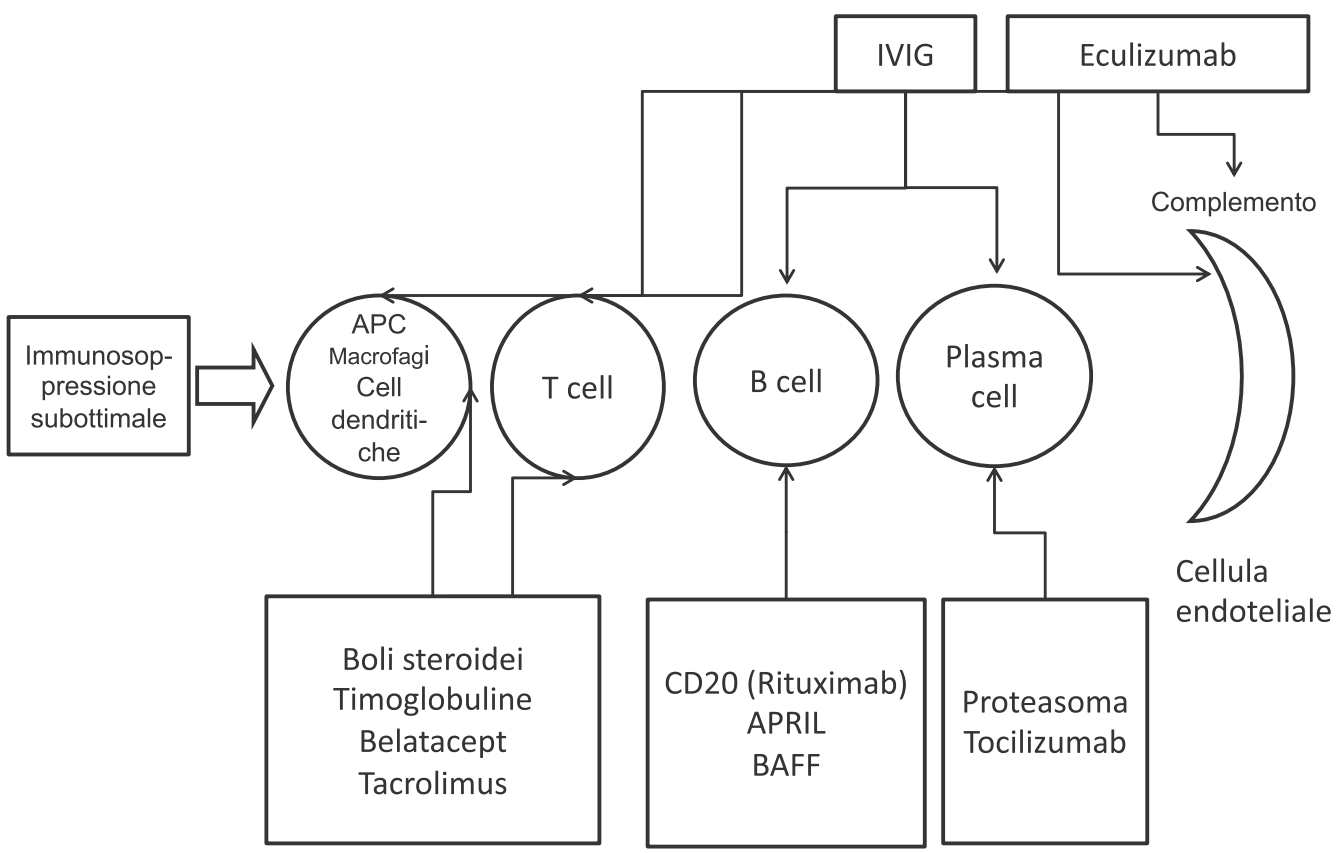

Fig. 1 - Multitrattamento del rigetto acuto anticorpo-mediato

Tabella 1 - Modalità di trattamento del AMR acuto

1) Rimozione o neutralizzazione di anticorpi: plasmaferesi, immunoassorbimento, immunoglobuline endovena, splenectomia

2) Terapie anti-cellule B: Micofenolato mofetil, Rituximab, immunoglobuline endovena, splenectomia

3) Terapia anti-plasmacellule: Bortezomib

4) Terapia anti-cellule T: Globuline antitimociti

5) Passaggio a terapia a base di Tacrolimus

6) Inibizione delle fasi terminali del complemento: Eculizumab

cellulare e incremento dell'apoptosi (16). Questa azione è particolarmente pronunciata nelle plasmacellule, per cui il farmaco è usato in forme di AMR resistenti agli altri trattamenti $(17,18)$.

Il suo ruolo nel trattamento del AMR è ancora discusso anche per gli importanti effetti collaterali, come neuropatie, disturbi gastro-enterici e disturbi ematologici.

\section{(f) Eculizumab}

Eculizumab è un anticorpo monoclonale umanizzato diretto contro la proteina complementare C5. Inibisce la conversione di $\mathrm{C} 5$ in $\mathrm{C} 5 \mathrm{~b}$, prevenendo la formazione del complesso che attacca la membrana (C5b-C9). Uno studio prospettico ha documentato una riduzione di AMR e una riduzione della glomerulopatia da trapianto a un anno (19).

\section{(g) Ruolo delle globuline antilinfocitarie (ATG)}

Le ATG non sono di per sé indicate nel trattamento di AMR. Tuttavia, il rigetto acuto cellulare spesso coesiste con il
AMR. Le globuline antitimocitarie, efficaci nel trattamento del rigetto acuto cellulare, hanno effetti benefici anche sul coesistente AMR. Probabilmente le ATG riducono lo stimolo esercitato dalle cellule $T$ sulle cellule $B$, portando a una riduzione di produzione anticorpale.

II rigetto cronico mediato da anticorpi (cAMR) è di più difficile trattamento, se si eccettua l'incremento dell'immunosoppressione.

Recentemente, farmaci monoclonali anti-recettore per I'interleuchina 6, come Tocilizumab e Clarakizumab, si sono dimostrati utili. II Tocilizumab è stato usato con successo in uno studio non controllato in pazienti con CAM R, DSA e glomerulopatia da trapianto (20).

Il Clarakizumab è, ora, oggetto di uno studio (IMAGINE) attualmente in fase 3 (21).

\section{Disclosures}

Conflict of interest: The authors declare no conflict of interest. Financial support: This research received no specific grant from any funding agency in the public, commercial, or not-for-profit sectors.

\section{Bibliografia}

1. Paul Terasaki, Kazuo Mizutani. Antibody mediated rejection: update 2006. Clin J Am Soc Nephrol. 2006;1(3):400-3.

2. Regele H, Böhmig GA, Habicht A, et al. Capillary deposition of complement split product $\mathrm{C} 4 \mathrm{~d}$ in renal allografts is associated with basement membrane injury in peritubular and glomerular capillaries: a contribution of humoral immunity to chronic allograft rejection. J Am Soc Nephrol. 2002;13(9):2371-80.

3. Cosio FG, Gloor JM, Sethi S, Stegall MD. Transplant glomerulopathy. Am J Transplant. 2008;8(3):492-6.

4. Zou Y, Stastny P, Süsal C, Döhler B, Opelz G. Antibodies against MICA antigens and kidney-transplant rejection. N Engl J Med. 2007;357(13):1293-300. 
5. Kekomäki S, Kyllönen L, Salmela K, Koskimies S, Kekomäki R. Platelet-specific alloantigens in cadaveric renal transplantation. A prospective study. Effect of HPA-5b mismatch in acute vascular rejection of renal allografts. Tissue Antigens. 2001;57(2):154-7.

6. Dragun D, Müller DN, Bräsen JH, et al. Angiotensin II type 1-receptor activating antibodies in renal-allograft rejection. $\mathrm{N}$ Engl J Med. 2005;352(6):558-69.

7. Lee CY, Lotfi-Emran S, Erdinc M, et al. The involvement of FcR mechanisms in antibody-mediated rejection. Transplantation. 2007;84(10):1324-34.

8. Solez K, Colvin RB, Racusen LC, et al. Banff 07 classification of renal allograft pathology: updates and future directions. Am J Transplant. 2008;8(4):753-60.

9. Colvin RB. Antibody-mediated renal allograft rejection: diagnosis and pathogenesis. J Am Soc Nephrol. 2007;18(4):1046-56.

10. Sis B, Jhangri GS, Bunnag S, Allanach K, Kaplan B, Halloran PF. Endothelial gene expression in kidney transplants with alloantibody indicates antibody-mediated damage despite lack of C4d staining. Am J Transplant. 2009;9(10):2312-23.

11. Böhmig GA, Wahrmann $\mathrm{M}$, Regele $\mathrm{H}$, et al. Immunoadsorption in severe C4d-positive acute kidney allograft rejection: a randomized controlled trial. Am J Transplant. 2007;7(1):117-21.

12. Becker YT, Becker BN, Pirsch JD, Sollinger HW. Rituximab as treatment for refractory kidney transplant rejection. Am J Transplant. 2004;4(6):996-1001.

13. Faguer S, Kamar N, Guilbeaud-Frugier C, et al. Rituximab therapy for acute humoral rejection after kidney transplantation. Transplantation. 2007;83(9):1277-80.
14. Kaposztas Z, Podder H, Mauiyyedi S, et al. Impact of rituximab therapy for treatment of acute humoral rejection. Clin Transplant. 2009;23(1):63-73.

15. Lefaucheur C, Nochy D, Andrade J, et al. Comparison of combination Plasmapheresis/IVIg/anti-CD20 versus high-dose IVIg in the treatment of antibody-mediated rejection. Am J Transplant. 2009;9(5):1099-107.

16. Sadaka B, Alloway RR, Woodle ES. Clinical and investigational use of proteasome inhibitors for transplant rejection. Expert Opin Investig Drugs. 2011;20(11):1535-42.

17. Everly MJ, Everly JJ, Susskind B, et al. Bortezomib provides effective therapy for antibody- and cell-mediated acute rejection. Transplantation. 2008;86(12):1754-61.

18. Walsh RC, Everly JJ, Brailey P, et al. Proteasome inhibitorbased primary therapy for antibody-mediated renal allograft rejection. Transplantation. 2010;89(3):277-84.

19. Stegall MD, Diwan $T$, Raghavaiah $S$, et al. Terminal complement inhibition decreases antibody-mediated rejection in sensitized renal transplant recipients. Am J Transplant. 2011;11(11): 2405-13.

20. Choi J, Aubert O, Vo A, et al. Assessment of Tocilizumab (AntiInterleukin-6 Receptor Monoclonal) as a Potential Treatment for Chronic Antibody-Mediated Rejection and Transplant Glomerulopathy in HLA-Sensitized Renal Allograft Recipients. Am J Transplant. 2017;17(9):2381-9.

21. IMAGINE (Interleukin 6 Blockade Modifying AntibodyMedited Graft Injury and eGFR Decline) Clin Gov. Identifier NCT03744910. 